\title{
Étude du comportement de deux variétés de soja dans le Nord de la France. Influence de la densité
}

\author{
I Lejeune-Henaut
}

INRA, laboratoire de génétique et d'amélioration des plantes, 80200 Estrees-Mons, France

(Reçu le 4 janvier 1991; accepté le 10 juin 1991)

\begin{abstract}
Résumé - Le comportement de 2 variétés de soja du groupe de maturité 000 a été étudié en 1988 et 1989, en conditions de plein champ dans le Nord de la France. Ces 2 génotypes se différencient par leur croissance indéterminée (Sito) ou semi-déterminée (Fiskeby). L'étude a été réalisée pour des peuplements de 40, 80 et 120 plantes par $\mathrm{m}^{2}$. La floraison et la croissance des fruits débute avant que la croissance de l'appareil végétatif ne soit terminée. Cette période de compétition pour les assimilats est plus longue chez Sito que chez Fiskeby. À 40 et 80 plantes par $\mathrm{m}^{2}$, la production en graines de Sito est supérieure à celle de Fiskeby. Dans ces conditions, le rendement est relié positivement à la quantité maximale de matière sèche végétative élaborée par le peuplement. Le passage de 40 à 80 plantes par $\mathrm{m}^{2}$ permet d'augmenter le rendement en produisant une plus grande quantité de matière sèche végétative, par unité de surface. À 120 plantes $/ \mathrm{m}^{2}$, la variété semi-déterminée Fiskeby a un rendement plus élevé que Sito. À cette densité, ni la matière sèche végétative maximale, ni l'indice de récolte ne différencient les 2 variétés. Les raisons de ce comportement sont donc à rechercher au niveau de la capacité du couvert végétal à produire des assimilats et à les transférer jusqu'aux grains.
\end{abstract}

Glycine max L Merrill = soja cv Sito et Fiskeby / densité / compétition / poids de matière sèche végétative / rendement en grain

Summary - Soybean behaviour in the north of France. Comparison between semi-determinate and indeterminate types and effects of plant population. The behaviour of 2 soybean varieties of maturity group 000 was studied in the north of France in 1988 and 1989 under field conditions. These 2 genotypes differ in their growth habit: indeterminate (Sito) and semi-determinate (Fiskeby). This experiment was conducted on populations of 40,80 and 120 plants $/ \mathrm{m}^{2}$. Blooming and fruit growth begin before vegetative growth is fully completed (figs 1,2 ). This competition period for assimilates is longer for Sito than for Fiskeby. At 40 and 80 plants per $m^{2}$, Sito grain production is more important than that of Fiskeby (fig 4). Under these conditions, grain yield is positively related to maximum vegetative dry weight (fig 3). The 80 plant/m² population allows a higher grain yield than the 40 plant/m ${ }^{2}$ by producing more vegetative dry weight on the same area basis (fig 3; table III). At 120 plants $/ \mathrm{m}^{2}$, the semi-determinate variety Fiskeby shows a higher grain yield than Sito. At this density, maximum vegetative dry weight and harvest index are the same for both genotypes (fig 3, tables III, IV) and cannot explain the yield difference. Reasons for this behaviour have to be investigated at the level of the canopy's capacity for assimilate production and translocation.

Glycine $\max$ L Merrill = soya cv Sito and Fiskeby / density / competition / vegetative dry weight / grain yiela 


\section{INTRODUCTION}

Les groupes de précocité du soja permettent de classer les cultivars en fonction de leurs besoins en température et en photopériode. Le groupe 000 , qui est le plus précoce, est représenté par des génotypes nécessitant moins de $1500^{\circ} \mathrm{C}$.j (seuil $6^{\circ} \mathrm{C}$ ) pour réaliser leur développement depuis le semis jusqu'à la maturité; les variétés qui le composent sont également peu sensibles à la durée du jour pour la mise à fleur.

Ces caractéristiques expliquent le choix de variétés du groupe 000 pour tester le comportement du soja en plein champ dans les conditions du Nord de la France.

Nous avons, d'autre part, voulu prendre en compte le type de croissance des plantes. La plupart des variétés cultivées en France sont de type indéterminé, mais certains travaux ont montré l'intérêt des types déterminés et semidéterminés dans des conditions de culture particulières.

Ces variétés ont tout d'abord été préconisées dans les zones de culture climatiquement favorables pour leur aptitude à valoriser des pratiques culturales intensives (irrigation, écartements réduits et peuplements élevés (Cooper, 1981; Beaver et Johnson, 1981; Chang et al, 1982; Blanchet et al, 1986). Lorsqu'elle est mise en évidence, cette aptitude est souvent reliée à la stature plus courte de ces variétés qui leur confère une meilleure résistance à la verse, et limite les pertes de grains à la récolte.

Un autre aspect du comportement de la plante, lié au type de croissance, mérite d'être considéré. II s'agit de la compétition entre puits végétatifs et reproducteurs.

Chez les variétés de soja de type indéterminé, l'allongement de la tige et la production de feuilles se poursuivent bien après le début de la floraison. Hanway et Weber (1971) ont montré que le poids de matière sèche de la tige est multiplié par 3 pendant la période de formation des gousses et des grains. Pendant le même temps, on observe une augmentation d'au moins 2 unités d'indice foliaire (Weber et al, 1966). Ces résultats suggèrent une forte compétition pour les assimilats entre puits végétatifs et reproducteurs, au moment de la formation des gousses et des grains. Cette compétition est sans doute d'autant plus élevée que le cycle est court (pas de rattrapage possible dans le temps) et que les assimilats sont peu abondants (rayonnement global limitant).
Les résultats de Vidal et Astruc (1984) montrent qu'il semble exister une compétition défavorable au rendement en grain entre la formation des nœuds pendant la phase reproductive et le remplissage des organes reproducteurs.

En suivant le devenir du ${ }^{14} \mathrm{CO}_{2}$ assimilé par une feuille, Grima-Pettenati et al (1987) ont effectivement mis en évidence un comportement différent entre 2 variétés déterminée et indéterminée : chez le type déterminé la migration des photosynthétats vers le haut de la plante (organes végétatifs en croissance) est moins importante que chez le type indéterminé et elle diminue beaucoup plus précocement, dès le stade R2 (fin floraison). Cette différence de comportement n'est toutefois pas mise en relation avec le rendement en grains.

D'autres travaux (Egli et al, 1985; Egli, 1988) montrent que la production grainière est plutôt reliée au nombre de nœuds et à la vitesse de croissance globale de la culture, qu'à l'allocation des assimilats entre parties végétatives et reproductrices.

Quelle que soit l'influence de la compétition entre les 2 types de puits, le carbone et l'azote sont redistribués à la fin du remplissage des graines depuis les parties végétatives de la plante jusqu'aux gousses (Egli et al, 1980; Zeiher et al, 1982). La sénescence du limbe s'accompagne d'une dégradation massive de protéines foliaires (Wittenbach et al, 1980; Franceschi et al, 1983) et l'azote ainsi libéré est transféré dans les graines (Warembourg et al, 1982). Il est possible que ce rôle de réservoir de l'appareil végétatif permette de compenser l'effet de la compétition entre organes végétatifs et reproducteurs en croissance.

L'objectif de cette étude est d'étudier le comportement de 2 variétés très précoces, l'une à croissance indéterminée et l'autre semidéterminée, dans des conditions extrêmes de culture. Nous sommes en particulier intéressés par la production grainière réalisée avec des peuplements variant de 40 plantes $/ \mathrm{m}^{2}$ (densité utilisée pour les groupes I et II en France) à 80 et 120 plantes $/ \mathrm{m}^{2}$ (peuplements supérieurs à ceux habituellement recommandés pour les variétés précoces).

Ce travail s'est effectué en suivant le déroulement des phases végétatives et reproductrices, et en comparant la matière sèche allouée aux différents organes, en cours de végétation et à la récolte. 


\section{MATÉRIEL ET MÉTHODES}

\section{Situation de l'essai}

L'expérimentation a été conduite en 1988 et 1989 à la station INRA d'Estrées-Mons (latitude : $50^{\circ} \mathrm{N}$ ) sur un sol limoneux profond. Les principales caractéristiques météorologiques des 2 années sont regroupées dans le tableau I.

\section{Matériel végétal}

Deux variétés ont été utilisées : l'une, Sito est de type indéterminé, et l'autre, Fiskeby est semi-déterminée. Toutes les 2 appartiennent au groupe de maturité 000 .

\section{Conduite agronomique}

Le semis a été réalisé le 28 avril en 1988 et le 5 mai en 1989, avec un semoir pneumatique. Trois densités ont été testées dans un dispositif en blocs : 40, 80 et 120 plantes $/ \mathrm{m}^{2}$. Chaque variété est répétée 4 fois dans des parcelles élémentaires de 6 rangs à écartement de $0,20 \mathrm{~m}$.

En raison d'une mauvaise levée de certaines parcelles de Fiskeby en 1989, seules les données concernant les densités 40 et 80 plantes $/ \mathrm{m}^{2}$ ont été exploitées pour cette variété.

Le sol n'ayant jamais porté de soja, une double inoculation du sol et de la graine (préalablement enrobée par un fongicide : Quinolate Pro) a été pratiquée.

Tableau I. Extrait des données météorologiques mensuelles de la station INRA d'Estrées-Mons, pour 1988 et 1989 .

\begin{tabular}{|c|c|c|c|c|c|}
\hline Mois & mai & juin & juillet & août & sept \\
\hline \multicolumn{6}{|c|}{ Température moyenne $\left({ }^{\circ} \mathrm{C}\right)$} \\
\hline $1988:$ & 14,0 & 15,2 & 16,3 & 17,5 & 14,2 \\
\hline $1989:$ & 14,8 & 15,5 & 18,7 & 17,7 & 15,9 \\
\hline \multicolumn{6}{|c|}{ Pluviométrie (mm) } \\
\hline 1988 : & 68,6 & 16,5 & 86,5 & 42,5 & 52,5 \\
\hline $1989:$ & 32,5 & 44,0 & 26,5 & 19,0 & 28,0 \\
\hline \multicolumn{6}{|c|}{ Rayonnement global (MJ/m²) } \\
\hline 1988 : & 575,4 & 554,9 & 560,8 & 526,9 & 280,6 \\
\hline $1989:$ & 704,7 & 675,2 & 639,6 & 527,8 & 359,2 \\
\hline
\end{tabular}

Le désherbage a été effectué en post semis avec les produits commerciaux Lasso et Ronstar aux doses respectives de 4 I et 1,5 l/ha. La repousse d'adventices à l'intérieur des parcelles a été négligeable, et facilement corrigée par un désherbage manuel.

En 1989, compte tenu des conditions climatiques, l'ensemble de l'essai a été irrigué à 6 reprises du 22 mai au 26 juillet, à raison de $20 \mathrm{~mm}$ pour les 5 premiers passages et de $30 \mathrm{~mm}$ pour le dernier. Cette irrigation a été réalisée à l'aide d'une rampe à aspersion.

\section{Observations et mesures}

En 1989, la progression de la floraison a été suivie sur 3 plantes individualisées dans chaque parcelle (soit 12 plantes pour chaque traitement) : à partir du début de la floraison, 3 observations hebdomadaires ont été faites de façon à dater la première fleur ouverte à chaque nœud de la tige principale. Les mêmes observations ont été faites sur les ramifications éventuelles.

En 1988, un suivi moins précis avait été réalisé : il nous a permis de dater le début et la fin de la période de floraison.

Au cours des 2 années, 6 prélèvements ont été effectués en cours de végétation, afin de suivre l'évolution de la matière sèche des différents organes végétatifs et reproducteurs. Ces données ont été recueillies sur:

- 5 plantes par parcelle élémentaire en 1988, soit 20 plantes au total par variété pour chaque densité (pesée individuelle des organes de chaque plante);

- 7 plantes par parcelle élémentaire en 1989, soit 28 plantes au total par variété pour chaque densité (pesée globale des organes des 7 plantes d'une même parcelle).

La récolte a été effectuée après la réalisation du stade de maturité physiologique (au moins $50 \%$ des plantes ayant les grains libres dans les gousses) le 27 septembre en 1988 et le $1^{\text {er }}$ septembre en 1989. II s'agit d'une récolte manuelle de :

- 25 plantes par parcelle élémentaire en 1988 ,

- 20 plantes par parcelle élémentaire en 1989.

Chacune des plantes récoltées a été l'objet des observations et mesures suivantes :

- caractéristiques morphologiques : hauteur totale, nombre de nœuds;

- poids de matière sèche des grains, poids de matière sèche des tiges et enveloppes des gousses.

Ces mesures ont été réalisées sur la tige principale et les ramifications. Elles ont permis de calculer, pour chaque plante, un indice de récolte (IR) qui représente le rapport du poids de matière sèche des grains sur le poids de matière sèche totale. 


\section{RÉSULTATS ET DISCUSSION}

\section{Développement et croissance}

Afin de ne pas surcharger les figures, seules les données concernant la densité 80 plantes $/ \mathrm{m}^{2}$ et l'année 1989 seront exposées dans ce paragraphe.

II faut cependant signaler que l'influence de la densité s'exprime sur :

- la durée de la phase levée-maturité : la maturité est atteinte plus rapidement $\left(55^{\circ} \mathrm{C}\right.$.j) pour les peuplements de 80 et 120 plantes $/ \mathrm{m}^{2}$ que pour la densité de 40 plantes $/ \mathrm{m}^{2}$;

- la date d'arrêt de la croissance végétative de Sito : pour les 2 années d'expérimentation, la croissance végétative (mesurée par l'allongement de la tige principale et par l'accroissement de matière sèche des tiges) a demandé en moyenne $180^{\circ} \mathrm{C}$.j (seuil $6^{\circ} \mathrm{C}$, depuis la levée) de plus chez Sito que chez Fiskeby, à 40 et 80 plantes $/ \mathrm{m}^{2}$ (fig 1). À 120 plantes $/ \mathrm{m}^{2}$, la croissance végétative de Sito s'arrête en même temps que celle de Fiskeby.

Le comportement aux basses et moyennes densités se traduit par une hauteur finale de la tige principale significativement plus grande pour Sito que pour Fiskeby (tableau II). Le nombre total de nœuds (1989) où l'allongement des entre-nœuds (1988) expliquent les différences de hauteur obtenues à maturité.

La floraison débute presque simultanément pour les 2 variétés (la différence, en nombre de j depuis la levée n'est pas significative, au seuil de $5 \%$ ), mais elle dure plus longtemps chez Sito (fig 1), quelle que soit la densité :
Tableau II. Hauteur finale et nombre de nœuds de la tige principale. Pour une année, les valeurs suivies par une même lettre ne sont pas significativement différentes (test de rang, Tukey, $\alpha=0,05$ ).

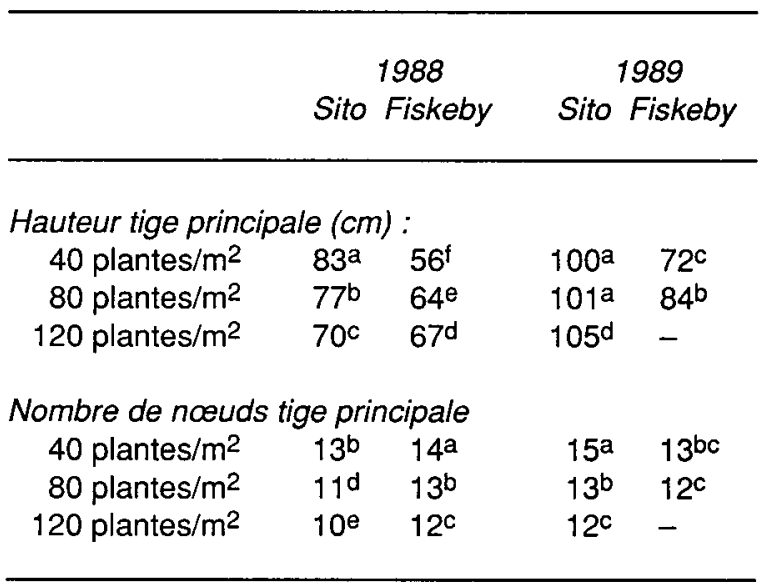

Le déroulement complet de cette phase nécessite $330^{\circ} \mathrm{C}$.j pour Sito et $190^{\circ} \mathrm{C}$.j pour Fiskeby qui se caractérise par une floraison groupée des derniers nœuds de la tige principale et de l'ensemble des ramifications (fig 2).

Ces premiers résultats nous permettent de constater un fonctionnement différent des 2 variétés :

- la période de compétition pour les assimilats entre organes végétatifs et reproducteurs est plus longue chez Sito que chez Fiskeby, pour les peuplements de 40 et 80 plantes $/ \mathrm{m}^{2}$.

- Fiskeby possède une phase reproductive (atlongement des gousses, remplissage et maturation des grains) plus longue; cette différence concerne essentiellement les organes fructifères des derniers nœuds de la tige principale et des ramifications.
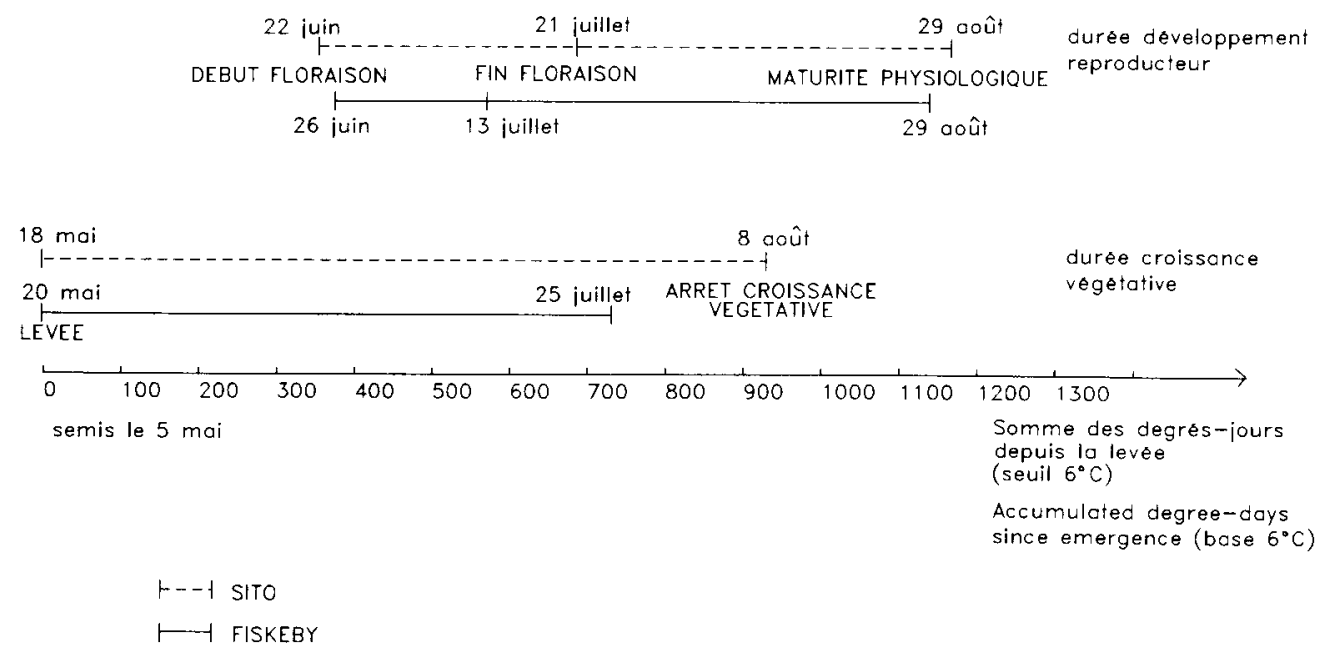

Fig 1. Durée des principales phases du développement et de la croissance en 1989 (densité : 80 plantes $/ \mathrm{m}^{2}$ ). 


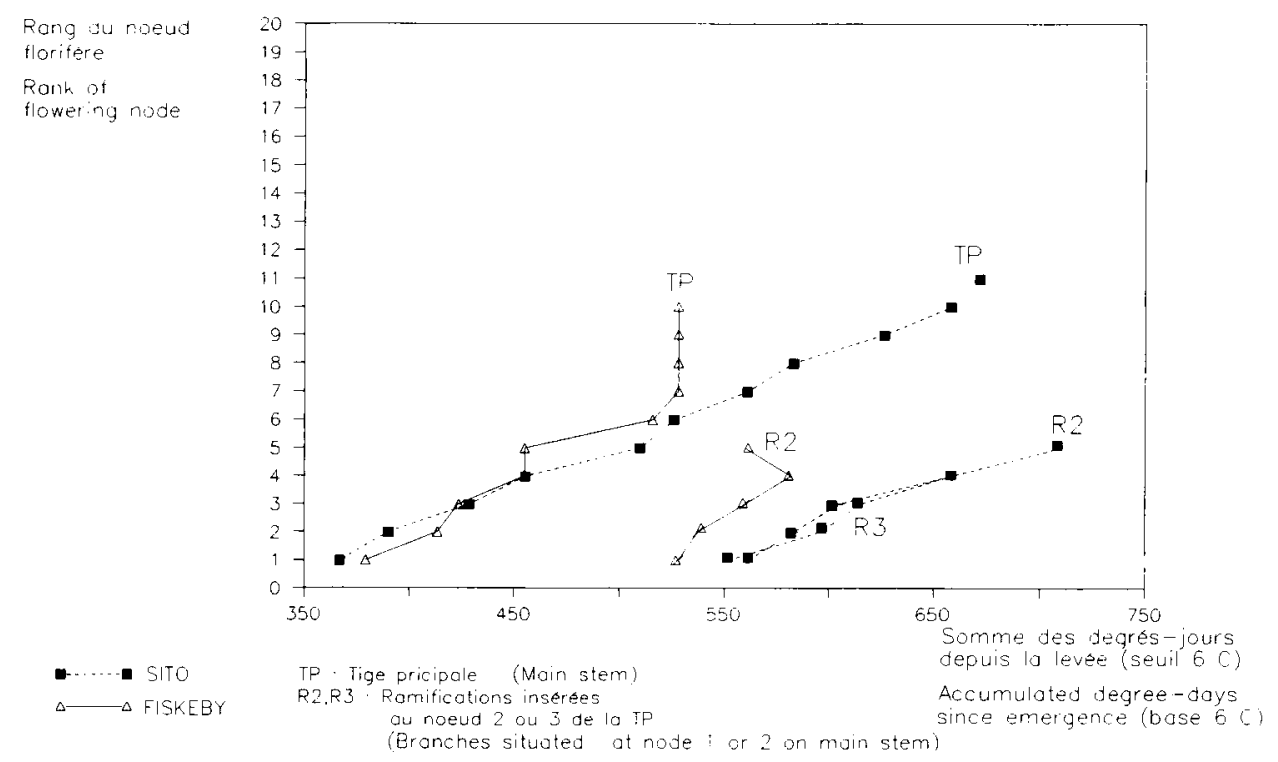

Fig 2. Déroulement de la floraison en 1989 sur la tige principale et les ramifications (densité : 80 plantes $/ \mathrm{m}^{2}$ ).

\section{Bilans de matière sèche végétative et reproductrice}

\section{Matière sèche végétative maximale}

Le tableau III regroupe les poids par organe lorsque le poids de matière sèche végétative de la plante est maximal (les plantes ont atteint les stades R5 à R6 de Fehr et al (1977).
Seuls les peuplements de 40 et 80 plantes $/ \mathrm{m}^{2}$ mettent en évidence des comportements différentiels, relatifs à l'accumulation de matière sèche des 2 génotypes.

La matière sèche allouée à l'ensemble des organes végétatifs d'une plante est plus importante pour Sito que pour Fiskeby.

Tableau III. Poids de matière sèche végétative maximale (environ $760^{\circ} \mathrm{C}$.j en $1988,900^{\circ} \mathrm{C}$.j en 1989) valeurs exprimées en $\mathrm{g} / \mathrm{plante}$.

\begin{tabular}{|c|c|c|c|c|c|c|c|c|c|c|c|c|}
\hline & \multicolumn{6}{|c|}{1988} & & & & \multirow{2}{*}{\multicolumn{3}{|c|}{$\frac{1989}{80 \mathrm{pl} / \mathrm{m}^{2}}$}} \\
\hline & \multicolumn{3}{|c|}{$40 \mathrm{pl} / \mathrm{m}^{2}$} & \multicolumn{3}{|c|}{$80 \mathrm{pl} / \mathrm{m}^{2}$} & \multicolumn{3}{|c|}{$120 \mathrm{pl} / \mathrm{m}^{2}$} & & & \\
\hline & Sito & \multicolumn{2}{|c|}{ Fiskeby } & Sito & \multicolumn{2}{|c|}{ Fiskeby } & Sito & \multicolumn{2}{|c|}{ Fiskeby } & Sito & \multicolumn{2}{|c|}{ Fiskeby } \\
\hline \multicolumn{13}{|l|}{ Axe principal : } \\
\hline tiges & 3,3 & 2,3 & $\left({ }^{*}\right)$ & 2,9 & 2,1 & $\left({ }^{*}\right)$ & 1,9 & 1,9 & (NS) & 4,8 & 3,9 & (NS) \\
\hline pétioles & 0,9 & 0,9 & (NS) & 0,8 & 0,8 & (NS) & 0,5 & 0,6 & (NS) & 1,1 & 1,3 & (NS) \\
\hline folioles & 3,4 & 2,9 & (NS) & 2,7 & 2,5 & (NS) & 1,8 & 1,9 & (NS) & 2,6 & 2,7 & (NS) \\
\hline total & 7,6 & 6,1 & $\left({ }^{*}\right)$ & 6,4 & 5,4 & $\left({ }^{*}\right)$ & 4,2 & 4,4 & (NS) & 8,5 & 7,9 & (NS) \\
\hline \multicolumn{13}{|l|}{ Ramifications : } \\
\hline tiges et & & & & & & & 0,3 & $\ll$ & (NS) & 1,4 & 0,1 & $(*)$ \\
\hline pétioles & 2,3 & 1,5 & $\left({ }^{*}\right)$ & 0,6 & & $\left({ }^{\star}\right)$ & 0,1 & $\ll$ & $\left({ }^{*}\right)$ & & & \\
\hline folioles & 1,7 & 1,7 & (NS) & 0,4 & 0,2 & (NS) & 0,1 & $\ll$ & $\left({ }^{*}\right)$ & 0,8 & 0,1 & (NS) \\
\hline total & 4,0 & 3,2 & (NS) & 1,0 & 0,5 & $\left({ }^{*}\right)$ & 0,2 & $\ll$ & $\left({ }^{*}\right)$ & 2,5 & 0,2 & $\left({ }^{*}\right)$ \\
\hline Plante entière : & 11,6 & 9,3 & $\left({ }^{*}\right)$ & 7,4 & 5,9 & $\left({ }^{*}\right)$ & 4,4 & 4,4 & (NS) & 11,0 & 8,1 & (NS) \\
\hline
\end{tabular}

« : valeur inférieure à $0,1 \mathrm{~g}$. L'effet variété est : (") : significatif (Student, $\alpha<=0,05$ ). Les différences significatives sont plus élevées en 1989, car les pesées ont été faites globalement pour chaque bloc et non pas plante à plante comme en 1988. 


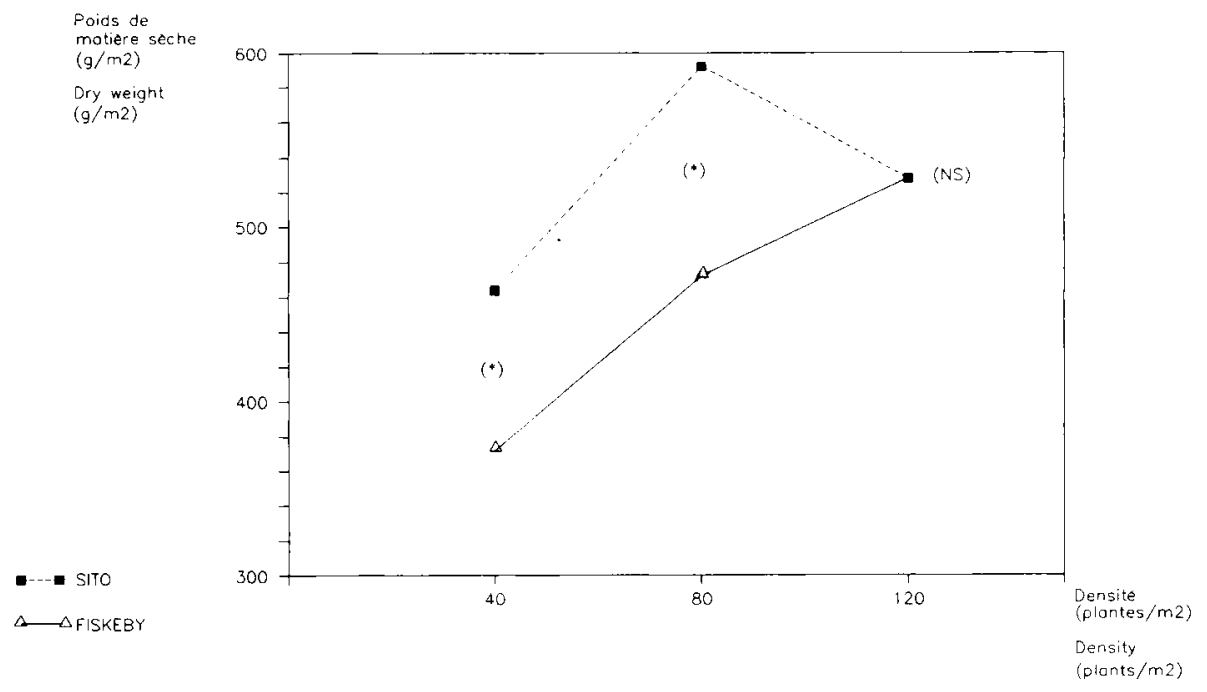

Fig 3. Poids de matière sèche végétative maximale (environ $760^{\circ} \mathrm{C}$.j en 1988). Valeurs exprimées en $\mathrm{g} / \mathrm{m}^{2}$. L'effet densité est significatif (Student, $\alpha \leq 0,05)$. Résultat du test de rang de Tukey $(\alpha=0,05): 40<80=120$. L'effet variété est : significatif (Student, $\alpha \leq 0,05):\left({ }^{*}\right)$; non significatif : (NS)

Cette différence est due à la matière sèche allouée aux tiges :

- au niveau des ramifications en 1989 ,

- au niveau de l'axe principal et des ramifications en 1988.

L'effet année est relativement important sur la matière sèche végétative produite par un même génotype : à 80 plantes $/ \mathrm{m}^{2}$, les valeurs de 1989 sont plus élevées de 49 et $37 \%$, par rapport à 1988, pour Sito et Fiskeby respectivement.
La production maximale de matière sèche par unité de surface est représentée à la figure 4 :

- les 2 variétés produisent une matière sèche végétative plus importante à 80 et 120 plantes $/ \mathrm{m}^{2}$;

- il existe une interaction significative $(\alpha=0,10)$ entre les effets variété et densité. L'analyse de l'effet variété pour chaque niveau de densité suggère que Fiskeby produit d'autant plus de matière sèche végétative par $\mathrm{m}^{2}$ que la densité de peuplement est élevée.

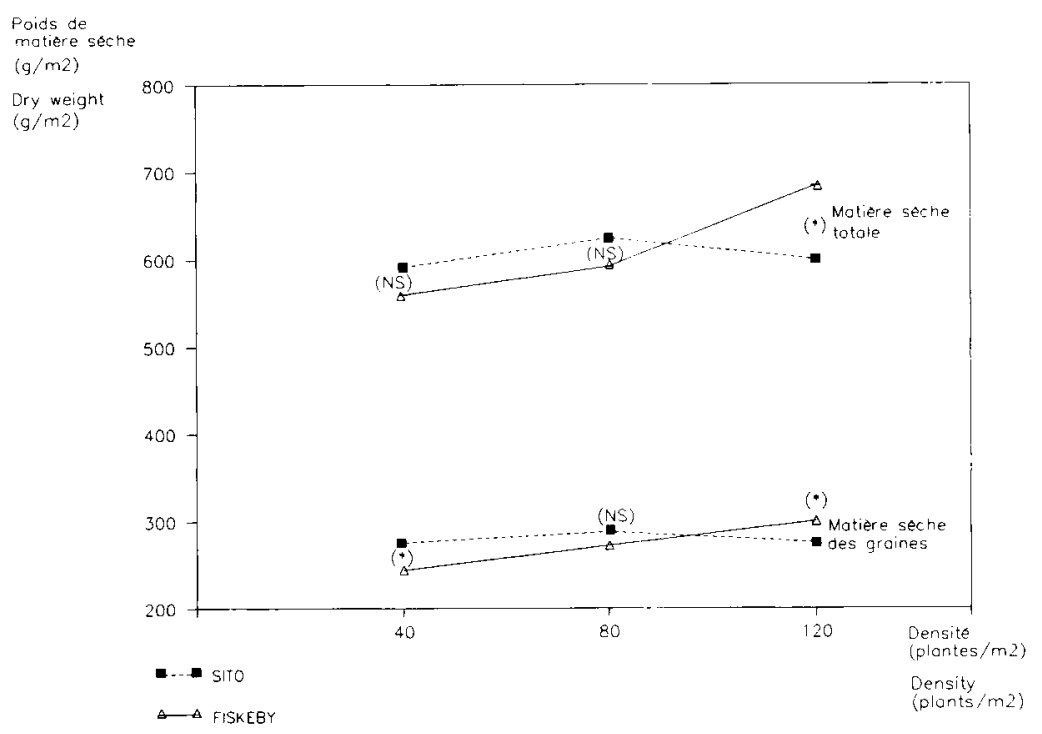

Fig 4. Poids de matière sèche totale et poids de matière sèche des grains à la récolte. L'effet variété est : $\left(^{*}\right)$ : significatif (Student, $\alpha \leq 0,05$ ); (NS) : non significatif. 


\section{Matière sèche totale à la récolte}

Le tableau IV contient les matières sèches reproductrices (grains) et résiduelles (tiges et enveloppes des gousses) de l'axe principal et des ramifications d'une plante.

La matière sèche individuelle à la récolte diminue lorsque la densité augmente. Cette baisse est due à la disparition progressive des ramifications et dans une moindre proportion à une production inférieure de la tige principale.

Sito s'avère le génotype le plus sensible aux variations des conditions de compétition : le poids de matière sèche des graines de la tige principale à 120 plantes $/ \mathrm{m}^{2}$ ne représente que $51 \%$ de celui obtenu au peuplement le plus faible, alors que cette proportion est de $61 \%$ pour Fiskeby. À 120 plantes $/ \mathrm{m}^{2}$, la production grainière de la tige principale de Sito est ainsi inférieure à celle de Fiskeby, ce qui, du fait de la non-compensation par les ramifications, explique sa moindre productivité.

La dernière ligne du tableau IV présente les valeurs de l'indice de récolte (IR). L'IR à 120 plantes par $\mathrm{m}^{2}$ est significativement plus faible qu'aux 2 autres densités (seuil de $5 \%$ ). Cette différence souligne l'importance de la compétition entre plantes d'un même peuplement pour la répartition finale des assimilats. Elle indique déjà une limite de gains de rendement que l'on peut espérer en augmentant la densité : l'augmentation du poids des grains au $\mathrm{m}^{2} \mathrm{n}^{\prime}$ est pas proportionnelle à l'augmentation du poids de matière sèche totale, quand elle existe.

L'absence de différence significative de I'IR entre la variété indéterminée et la variété semidéterminée montre que ce n'est pas par l'allocation finale des assimilats entre grain et "paille" que le comportement des 2 variétés se différencie.

La figure 4 représente la matière sèche totale récoltée par $\mathrm{m}^{2}$ et la matière sèche de grains par $\mathrm{m}^{2}$ en 1988. II y a une interaction significative entre variété et densité pour ces 2 variables. La production augmente pour les 2 variétés de 40 à 80 plantes $/ \mathrm{m}^{2}$. À 120 plantes par $\mathrm{m}^{2}$, elle continue d'augmenter pour Fiskeby au contraire de Sito.

\section{Relation entre matière sèche végétative maximale et rendement en grain}

\section{Densités 40 et 80 plantes $/ \mathrm{m}^{2}$}

D'une façon générale, nous avons remarqué que pour ces densités, les 2 variétés produisent d'autant plus de matière sèche de grains qu'elles ont élaboré de matière sèche végétative. Ces résultats sont en accord avec ceux de Egli et al (1985) et Egli (1988).

Tableau IV. Répartition de la matière sèche totale à la récolte.

\begin{tabular}{|c|c|c|c|c|}
\hline \multicolumn{3}{|c|}{1988} & \multicolumn{2}{|c|}{1989} \\
\hline $\begin{array}{c}40 \\
\text { plantes } / m^{2}\end{array}$ & $\begin{array}{c}80 \\
\text { plantes } / m^{2}\end{array}$ & $\begin{array}{c}120 \\
\text { plantes } / m^{2}\end{array}$ & $\begin{array}{c}40 \\
\text { plantes } / m^{2}\end{array}$ & $\begin{array}{c}80 \\
\text { plantes } / m^{2}\end{array}$ \\
\hline Sito Fiskeby & Sito Fiskeby & Sito Fiskeby & Sito Fiskeby & Sito Fiskeby \\
\hline
\end{tabular}

\begin{tabular}{|c|c|c|c|c|c|c|c|c|c|c|c|c|c|c|c|}
\hline \multicolumn{16}{|c|}{ Matière sèche grains $(g)$ : } \\
\hline tige principale & 4,1 & 4,1 & (NS) & 3,0 & 3,3 & $(*)$ & 2,1 & 2,5 & $\left({ }^{*}\right)$ & 7,2 & 6,4 & $\left(*^{*}\right)$ & 5,6 & 5,5 & (NS) \\
\hline ramification & 2,9 & 2,1 & $(*)$ & 0,7 & 0,2 & $\left({ }^{*}\right)$ & 0,2 & $\ll$ & $(*)$ & 5,9 & 1,5 & $(*)$ & 1,2 & $\ll$ & $\left({ }^{*}\right)$ \\
\hline totale & 6,9 & 6,2 & $\left({ }^{*}\right)$ & 3,7 & 3,5 & (NS) & 2,3 & 2,6 & $(*)$ & 13,0 & 7,9 & $(*)$ & 6,8 & 5,5 & $\left({ }^{*}\right)$ \\
\hline \multicolumn{16}{|c|}{ Matière sèche résiduelle $(g)$} \\
\hline tige principale & 4,9 & 5,8 & (NS) & 3,6 & 3,9 & $\left({ }^{*}\right)$ & 2,6 & 3,0 & $\left({ }^{*}\right)$ & 8,8 & 6,9 & $(*)$ & 6,4 & 6,5 & (NS) \\
\hline ramification & 3,5 & 2,3 & $(*)$ & 0,6 & 0,2 & $(*)$ & 0,1 & 0,1 & (NS) & 6,4 & 1,7 & $\left({ }^{*}\right)$ & 1,5 & $\ll$ & $(*)$ \\
\hline totale & 8,0 & 8,1 & (NS) & 4,2 & 4,1 & (NS) & 2,7 & 3,1 & $(*)$ & 15,1 & 8,6 & $(*)$ & 7,9 & 6,5 & $\left(*^{*}\right)$ \\
\hline Matière sèche totale & 14,9 & 14,3 & (NS) & 7,9 & 7,6 & (NS) & 5 & 5,7 & $(*)$ & 28,2 & 16,5 & $\left({ }^{*}\right)$ & 14,8 & 12,0 & $(")$ \\
\hline IR : & 0,46 & 0,47 & (NS) & 0,47 & 0,46 & (NS) & 0,45 & 0,45 & (NS) & 0,46 & 0,48 & (NS) & 0,46 & 0.45 & (NS) \\
\hline
\end{tabular}


La comparaison variétale est à l'avantage de la variété indéterminée Sito. En pleine végétation, celle-ci se différencie de Fiskeby essentiellement par un poids plus grand de matière sèche des tiges. Par contre, la matière sèche allouée aux organes photosynthétiques est équivalente pour les 2 variétés, quelles que soient l'année et la densité.

Deux hypothèses peuvent être envisagées :

- à matière sèche foliaire égale, le rendement photosynthétique de Sito est meilleur que celui de Fiskeby : les facteurs pouvant intervenir dans la détermination de ce rendement (taux de photosynthèse par unité de surface foliaire, durée de la photosynthèse, autoombrage, quantité d'assimilats transférés, etc) restent à préciser;

- la possibilité de remobilisation de réserves à partir des tiges est plus importante chez Sito.

D'une année sur l'autre, les conditions du milieu peuvent influer sur la matière sèche végétative produite, et par conséquent sur le rendement. L'avantage observé en 1989 pour le poids maximal de matière sèche végétative et le rendement des 2 variétés peut être en partie expliqué par les conditions environnementales. II est très vraisemblable que le rayonnement global plus élevé ainsi que l'irrigation appliquée cette année là, aient permis un développement plus important de l'appareil végétatif et pour finir un meilleur rendement.

\section{Densité 120 plantes $/ m^{2}$}

Lorsque les conditions de compétition à l'intérieur du peuplement deviennent très fortes, le comportement des 2 variétés, en ce qui concerne l'utilisation de leur potentiel végétatif, diffère. La matière sèche végétative maximale produite par les 2 génotypes est équivalente, par contre la matière sèche totale récoltée et le rendement en grains sont plus importants pour la variété semi-déterminée (fig 4).

Le couvert végétal de Fiskeby a donc un meilleur fonctionnement que celui de Sito à cette densité, mais les raisons peuvent en être diverses : photosynthèse plus importante, moins de pertes d'assimilats par respiration ou allocation au système racinaire, réallocation plus efficace des feuilles vers les grains au moment de la sénescence du feuillage. Ces hypothèses ne peuvent pas être vérifiées avec les mesures que nous avons effectuées.

\section{CONCLUSION}

Les données recueillies au cours de ces 2 années d'expérimentation nous ont permis, avant tout, de caractériser le comportement de variétés très précoces (groupe 000) dans les conditions climatiques du Nord de la France.

Les principaux enseignements que nous en avons tiré sont les suivants :

- la compétition entre organes végétatifs et reproducteurs existe, dans le sens où la croissance des organes reproducteurs commence avant que la croissance de l'appareil végétatif en soit terminée. Cette période de compétition est plus longue chez Sito que chez Fiskeby à 40 et 80 plantes $/ \mathrm{m}^{2}$. Pour ces peuplements, le rendement grainier de Sito reste supérieur ou égal à celui de Fiskeby. II faudrait toutefois comparer des lignées quasi isogéniques de Sito (différant seulement pour le type de croissance) pour savoir dans quelle mesure la durée de la période de compétition ne pénalise pas le rendement;

- la production en grains évolue dans le même sens que la production de matière sèche végétative maximale même si le coefficient de proportionnalité entre ces 2 variables diffère suivant la densité. La matière sèche végétative doit donc être considérée comme un potentiel utilisable à long terme, puisqu'elle est réallouée en partie aux grains pendant la phase finale de remplissage;

- l'augmentation de peuplement de 40 à 80 plantes $/ \mathrm{m}^{2}$ permet de compenser la baisse de production individuelle;

- à 120 plantes $/ \mathrm{m}^{2}$, la variété semi-déterminée Fiskeby produit un meilleur rendement en grain. Des précisions restent à apporter quant aux raisons de cette meilleure efficacité, car ni la matière sèche végétative maximale, ni l'indice de récolte ne différencient alors les 2 variétés.

L'effet d'interaction entre variété et densité est à prendre en compte dans les zones où les conditions climatiques conduisent à utiliser des peuplements élevés.

\section{REMERCIEMENTS}

Je remercie A Blake, $M$ Eteve, $M C$ Leforestier et $M$ Niarquin pour leur collaboration à la réalisation du manuscrit. 
Je remercie Messieurs R Bonhomme, $M$ Derieux et $P$ Roumet pour leur lecture critique de ce travail.

\section{RÉFÉRENCES}

Beaver JS, Johnson RR (1981) Response of determinate and indeterminate soybeans to varying cultural practices in the northern USA. Agron J73, 833-838

Blanchet R, Bouniols A, Constant S, Gelfi N, Marty JR, Puech J (1986) Biologie et formation du rendement de sojas déterminés et indéterminés en bonnes conditions culturales. Eurosoya 4, 10-17

Chang JF, Green DE, Shibles R (1982) Yield and agronomic performance of semideterminate and indeterminate soybean stem types. Crop Sci 22, 97 101

Cooper RL (1981) Development of short-statured soybean cultivars. Crop Sci 21, 127-131

Egli DB (1988) Alterations in plant growth and dry matter distribution in soybean. Agron J 80, 86-90

Egli DB, Guffy RD, Cheniae A (1980) Carbohydrate levels in soybean leaves during reproductive growth. Crop Sci 20, 468-473

Egli DB, Guffy RD, Leggett JE (1985) Partitioning of assimilate between vegetative and reproductive growth in soybean. Agron J 77, 917-922

Fehr WR, Caviness CE (1977) Stages of soybean development. lowa State Univ Ames, Spec Rep, 11 p
Franceschi VR; Wittenbach VA, Giaquinta RT (1983) Paraveinal mesophyll of soybean leaves in relation to assimilate transfer and compartmentation. Plant Physiol 72, 586-589

Grima-Pettenati J, Bailly-Fenech G, Latche JC (1987) Étude comparative des migrations d'assimilats chez deux variétés de soja, de type déterminé ou indéterminé. Influence d'une carence en azote. Agronomie 7, 447-456

Hanway JJ, Weber CR (1971) Dry matter accumulation in soybean (Glycine max L Merrill) plants as influenced by N, P and K fertilization. Agron $J 63$, 263-266

Vidal A, Astruc C (1984) Étude comparative de la croissance et du développement de quelques variétés de soja. Eurosoya 2, 39-46

Warembourg FR, Montange D, Bardin R (1982) The simultaneous use of ${ }^{14} \mathrm{CO}_{2}$ and ${ }^{15} \mathrm{~N}_{2}$ labelling techniques to study the carbon and nitrogen economy of legumes grown under natural conditions. Physiol Plant 56, 46-55

Weber CR, Shibles RM, Byth DE (1966) Effect of plant population and row spacing on soybean development and production. Agron J 58, 99-102

Wittenbach VA, Ackerson RC, Giaquinta RT, Hebert RR (1980) Changes in photosynthesis, ribulose biphosphate carboxylase, proteolytic activity and ultrastructure of soybean leaves during senescence. Crop Sci 20, 225-231

Zeiher C, Egli DB, Leggett JE, Reicosky DA (1982) Cultivar differences in $\mathrm{N}$ redistribution in soybeans. Agron J 14, 375-379 